\title{
Treatment patterns and overall survival in metastatic non-small-cell lung cancer in a real-world, US setting
}

\author{
Jason C Simeone*,1, Beth L Nordstrom¹, Ketan Patel ${ }^{2}$ \& Alyssa B Klein ${ }^{3}$ \\ ${ }^{1}$ Real-World Evidence, Evidera, Waltham, MA 02451, USA \\ ${ }^{2}$ Teradata UK Ltd, London, UK \\ ${ }^{3}$ Oncology Business Unit, AstraZeneca, Gaithersburg, MD 20878, USA \\ *Author for correspondence: Tel.: +1 781960 0274; Fax: +1 781767 0147; jason.simeone@evidera.com
}

\begin{abstract}
Aim: To conduct a retrospective analysis of electronic medical record data to understand real-world treatment patterns and overall survival (OS) in patients with metastatic non-small-cell lung cancer (NSCLC). Materials \& methods: We included $n=9656$ adults ( $\geq 18$ years) with metastatic NSCLC and no prior therapy. Data from 1 January 2013 to 31 January 2017 were analyzed. Results: Carboplatin plus paclitaxel was the most common first-line therapy (18.6\%), and nivolumab was the most common second- $(31.0 \%)$ and thirdline (38.4\%) therapy; $26.7 \%$ of all patients were untreated. Median OS from initial metastatic diagnosis was 11.1 months (95\% Cl: 10.8-11.5). Second-line immunotherapy extended OS by over 3 months versus second-line chemotherapy. Conclusion: Platinum-based therapy was the most common first-line therapy, and immunotherapy was the most common second- and third-line therapy. Median OS of patients with metastatic NSCLC was $<1$ year.
\end{abstract}

First draft submitted: 27 June 2019; Accepted for publication: 12 August 2019; Published online: 9 September 2019

Keywords: carcinoma • chemotherapy • electronic health records • immunotherapy • non-small-cell lung

Lung cancer is the leading cause of cancer-related deaths in the USA, with approximately 234,030 new cases and 154,050 lung cancer-related deaths in the USA in 2018 [1]. Between 80 and 85\% of patients with lung cancer have non-small-cell lung cancer (NSCLC) [1], which is characterized histologically as squamous cell carcinoma or nonsquamous cell carcinoma (adenocarcinoma and large-cell carcinoma) [2,3]. Stage IV NSCLC is the most advanced form of the disease, in which the cancer has metastasized to the pleura and beyond the lungs, and 55\% of patients have distant metastases at the time of initial NSCLC diagnosis in the USA [4]. The 5-year survival rate for stage IVA and IVB NSCLC (referred to as metastatic NSCLC, hereafter) is approximately 10 and $<1 \%$, respectively [5].

Platinum-based doublets have traditionally been the standard of care for patients with metastatic NSCLC [6,7], although these treatments have been associated with toxicity and only limited survival benefit $[8,9]$. In addition to platinum-based chemotherapy, newer treatments are now available, including targeted therapy and immunotherapy, either alone or in combination with standard chemotherapy [6,7]. Accordingly, the metastatic NSCLC treatment paradigm has evolved into a biomarker-driven personalized care model [10], with the PD-1/PD-L1 pathway recognized as an important immune checkpoint used by tumor cells (TC) to inhibit antitumor responses [11]. In the second- and later-line settings, nivolumab, pembrolizumab and atezolizumab were approved in the USA in March 2015, October 2015 and October 2016, respectively, for patients with metastatic NSCLC whose disease has progressed on/after platinum-based chemotherapy [12-14]. Pembrolizumab is approved as a single agent for the treatment of patients with metastatic NSCLC with disease progression on or after platinum-containing chemotherapy, whose tumors express PD-L1 (tumor proportion score $\geq 1 \%$ ) as determined by a US FDA-approved test [12]. In October 2016, pembrolizumab was approved in the USA as first-line treatment for patients with metastatic NSCLC with no EGFR mutations (EGFRm) or $A L K$ rearrangements who have $\geq 50 \%$ of TC expressing PD-L1, as determined using the FDA-approved Dako immunohistochemistry PD-L1 22C3 pharmDx companion

Future Medicine 
assay [15]. Furthermore, immunotherapy plus chemotherapy combination regimens are approved for patients with unselected metastatic NSCLC [12,14].

Due to the poor prognosis of patients with metastatic NSCLC and the limited availability of effective treatments, clinical research on treatment options such as immunotherapy must continue. Currently, dual blockade of the CTLA-4 and PD-1/PD-L1 pathways using immunotherapies is being explored in metastatic NSCLC [16-24]. Durvalumab + tremelimumab combination regimens have shown encouraging tolerability and clinical activity in a Phase Ib study [16], and the combination of nivolumab + ipilimumab as first-line therapy improved progression-free survival (PFS) in patients with metastatic NSCLC with a high tumor mutational burden (TMB), in both PD-L1 TC $<1$ and $\geq 1 \%$ subgroups [17]. In the Phase III MYSTIC trial (NCT02453282), the combination of durval$\mathrm{umab}+$ tremelimumab did not statistically significantly improve overall survival (OS) or PFS vs. chemotherapy in patients with PD-L1 TC $\geq 25 \%$ [18-20]. However, in exploratory analyses, the combination showed clinical activity in patients with high blood TMB (bTMB), with improvements in OS observed at bTMB thresholds from $\geq 12$ to $\geq 20 \mathrm{mut} / \mathrm{Mb}[20,21]$.

To complement the emergence of immunotherapy in metastatic NSCLC [12-25], it is important to understand the effectiveness of newer treatments and determine further treatment needs. Specifically, there remains an unmet need for real-world evidence documenting treatment patterns and survival outcomes following the approval and uptake of immunotherapy into the metastatic NSCLC setting. Therefore, the aim of the current retrospective study was to provide a historical perspective of real-world treatment patterns, clinical characteristics and OS in patients with previously untreated, metastatic NSCLC with no EGFRm or $A L K$ rearrangements, treated in US community oncology clinics around the time of the introduction of immunotherapy for metastatic NSCLC.

\section{Materials \& methods \\ Database description}

In this retrospective, observational study, anonymized electronic medical record (EMR) data from the Flatiron Health database [26] were analyzed. The database includes abstracted data from unstructured documents (e.g., medical notes) and structured data (e.g., laboratory results and treatment information). At the time of analysis, data were available for $>1.6$ million active patients from $>260$ community oncology clinics in the USA. Flatiron data are extracted through technology-enabled chart abstraction, generating datasets that allow a valid look at real-world treatment patterns and survival outcomes among patients with metastatic NSCLC $[8,27,28]$.

Study data complied with US patient confidentiality requirements. As the study used only existing de-identified patient records, Institutional Review Board approval and patient informed consent were not required.

\section{Patients}

The study population was intended to mirror the types of patients who are recruited into clinical trials of immunotherapy, in order to provide a historical, real-world cohort for comparison. Eligible patients for this study were $\geq 18$ years old with a diagnosis of histologically or cytologically documented metastatic NSCLC between 1 January 2013 and 31 January 2016, and no prior therapy for advanced or metastatic NSCLC. Patients were selected from a Flatiron cohort of patients with advanced NSCLC and included if they were initially diagnosed with metastatic (stage IV) disease, or if they had progressed to metastatic disease from an earlier stage; progression to metastatic disease was identified using International Classification of Diseases (ICD)-9 and ICD-10 codes indicating secondary tumors. Where recorded in their medical records, patients with $E G F R \mathrm{~m}$ and $A L K$ rearrangements were excluded; however, most patients did not have molecular test results. Data from 1 January 2013 to 31 January 2017 were extracted from the EMR. The date of first diagnosis with metastatic NSCLC was considered the index date. Patients were followed longitudinally until death or their last visit prior to the end of the study period, and were required to contribute a minimum of 1 day of follow-up. Patients were excluded if they had been part of clinical trials, had other malignant neoplasms (except nonmelanoma skin cancer and carcinoma in situ) before the index date, mixed small-cell lung cancer/NSCLC histology, NSCLC histology not otherwise specified, brain metastases or spinal cord compression. Patients with certain comorbid conditions, including autoimmune disease (e.g., rheumatoid arthritis and systemic lupus erythematosus), bleeding diathesis, hepatitis B or C, HIV, congestive heart failure, unstable angina or inflammatory bowel disease (e.g., Crohn's disease and ulcerative colitis), were also excluded. 


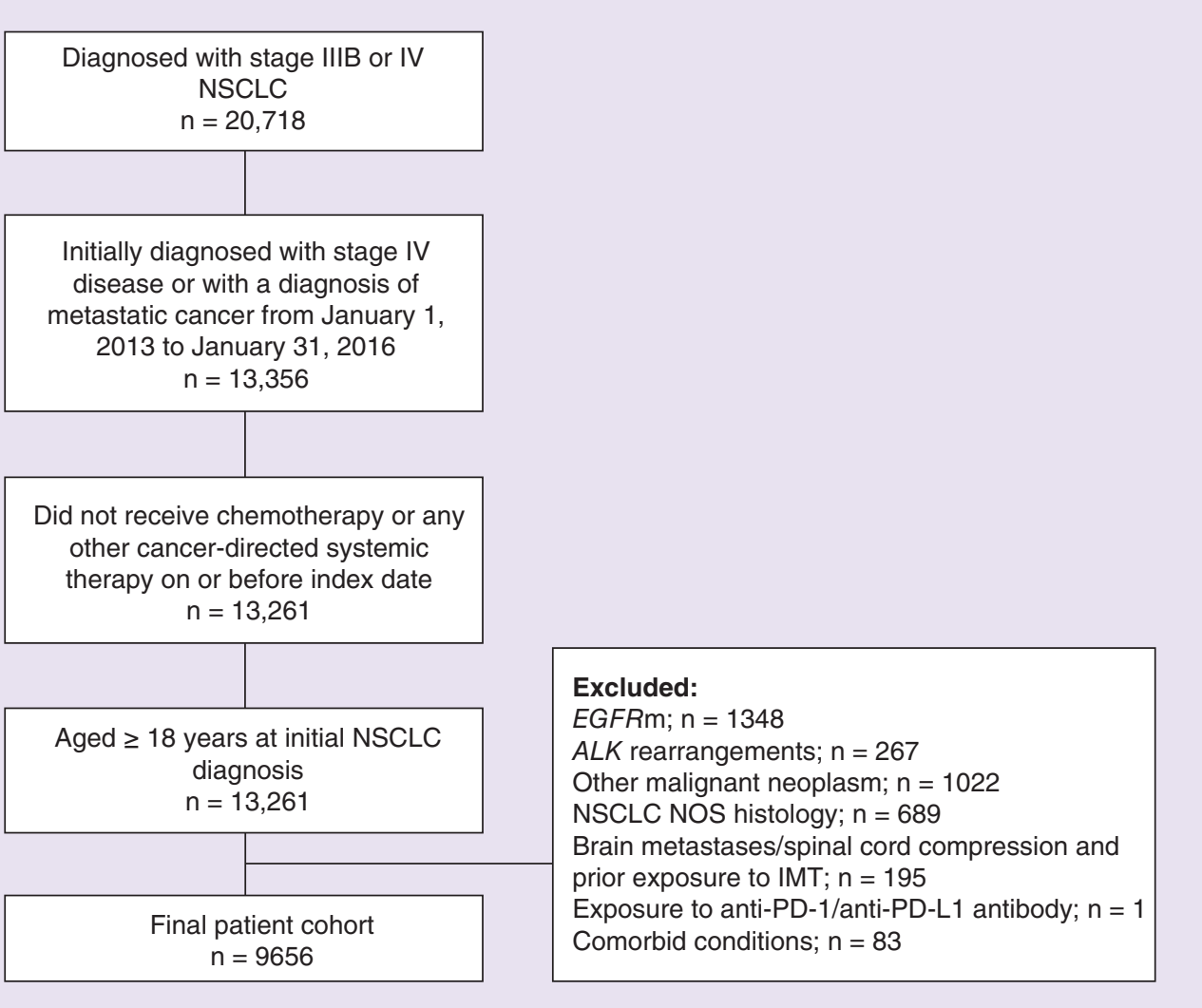

Figure 1. Study flow diagram.

EGFRm: EGFR mutation; IMT: Immunomodulatory therapy; NOS: Not otherwise specified; NSCLC: Non-small-cell lung cancer.

\section{Study end points}

Where available, baseline demographic and clinical characteristics, such as smoking history, comorbidities, disease stage at initial diagnosis and time from initial NSCLC diagnosis to the index date, were extracted from the EMR. Additional variables and covariates extracted at baseline and follow-up included metastatic sites and date(s) of diagnosis, Eastern Cooperative Oncology Group performance status (ECOG PS), biomarker status (EGFRm, ALK rearrangements, KRAS mutations and PD-L1 expression status) and concomitant supportive care drugs, where available.

Treatment patterns extracted were the type/class of therapy in all treatment lines, categorized into chemotherapy/nonimmunotherapy (platinum chemotherapy, other chemotherapy, targeted therapy or antiangiogenic therapy) or immunotherapy. The chemotherapy/nonimmunotherapy category was defined as any regimen containing only chemotherapy/nonimmunotherapy agents and did not need to include platinum-based chemotherapy. The immunotherapy category was defined as any regimen containing immunotherapy and could include platinum-based chemotherapy if taken in combination with immunotherapy. Other information extracted included the line of therapy (first, second and third or higher [referred to as third line, hereafter]), total number and duration of treatment lines (duration defined as start date of the line of treatment to the last recorded date of administration recorded in the Flatiron database), time from the index date to first-line treatment and time between treatment lines. OS was evaluated from the index date, date of initial NSCLC diagnosis, start of first-line therapy, by type of first-line treatment received and by treatment sequence in first- and second-line therapy.

\section{Statistical analysis}

The patient sample was a convenience population of all patients in the Flatiron database who met inclusion and exclusion criteria during the study period; no a priori power analyses were conducted. Study measures (i.e., patient demographic information, clinical characteristics and treatment patterns) were summarized with descriptive 
statistics. Mean, standard deviation (SD), median and range were reported for continuous measures. Categorical measures were summarized using frequencies and percentages. Kaplan-Meier methods were used to present OS in days; the follow-up period was divided into 10-month increments to indicate the number of patients still alive at each time point. OS data maturity was calculated as the number of deaths divided by the number of patients eligible at the start of each analysis. Median OS was calculated along with two-sided 95\% CIs. OS was also evaluated by the type of treatment received in each line of therapy using Kaplan-Meier curves. Statistical analyses were conducted using SAS ${ }^{\circledR}$ Enterprise Guide (NC, USA), and statistical tests were two-sided with a significance level of 0.05 .

\section{Results}

Baseline characteristics

A total of 13,261 adult patients with previously untreated stage IV disease were identified. Following the exclusion of patients who did not meet eligibility criteria, 9656 patients were included in the analysis (Figure 1). At baseline (defined as prior to or at the index date), the mean (SD) age of patients was $67.8(10.0)$ years, $54.5 \%$ were men and $62.3 \%$ were white; information on race was missing for $16.5 \%$ of patients (Table 1). Patients were distributed across the USA, and most patients (88.2\%) had a history of smoking. The majority of patients $(96.2 \%)$ were initially diagnosed with metastatic disease; $3.4 \%$ of patients were initially diagnosed with stage I-IIIB disease and progressed to metastatic disease (Table 1). The mean (SD) time from initial NSCLC diagnosis to first metastatic diagnosis for the overall cohort of patients (i.e. all patients diagnosed with metastatic disease and not just those initially diagnosed with early stage disease) was 14.6 (83.5) days. Metastatic sites were identified through secondary tumor ICD-10 codes that were not reported for $95.3 \%$ of patients; however, among the known metastatic sites $(n=451)$, more than half $(53.0 \%)$ of the patients had bone metastases (Table 1$)$.

\section{Clinical characteristics}

The median duration of follow-up was 253 days. The number of known metastatic sites increased during the followup period; among patients with a known metastatic site $(\mathrm{n}=4502)$, bone metastasis was the most common $(46.2 \%$; Table 2). During the follow-up period, PD-L1 testing rates increased from baseline $(<1-5.7 \%$, respectively), but remained low (Table 2). Few patients used concomitant supportive care drugs at baseline, and their use increased during the follow-up period; antiemetics were the most common (64.2\%), followed by solution fluids (41.8\%; Table 2). Among patients with recorded data on ECOG PS $<60$ days after index date $(\mathrm{n}=3600), 32.3 \%$ had an ECOG PS of 0; 39.3 and 20.5\% had an ECOG PS of 1 and 2, respectively; few patients had an ECOG PS of 3 or 4 (Table 2).

\section{First-line treatment patterns}

The median time from diagnosis to start of first-line therapy was 37 (range: -7 to 1459) days. The most common firstline regimens were carboplatin + paclitaxel and carboplatin + pemetrexed (18.6 and 16.6\%, respectively [Table 3]). Overall, 392 (32.6\%), $367(30.6 \%)$ and $442(36.8 \%)$ patients received $\leq 3,4-6$ and $\geq 7$ administrations of carboplatin + paclitaxel, respectively, and 569 (53.1\%), 414 (38.7\%) and 88 (8.2\%) patients received $\leq 3,4-6$ and $\geq 7$ administrations of carboplatin + pemetrexed, respectively. The median duration of first-line treatment was 64 (range: 1-1576) days.

\section{Second-line treatment patterns}

A median interval of 35 (range: 1-1301) days passed between first- and second-line therapy, and the median duration of second-line therapy was 69 (range: 1-1398) days. The most common second-line regimens were nivolumab (31.0\%; 241 [26.2\%], 214 [23.3\%] and 465 [50.5\%] patients received $\leq 3,4-6$ and $\geq 7$ administrations, respectively), pemetrexed $(9.4 \%)$ and docetaxel $(8.4 \%)$ (Table 3$)$. The median duration of nivolumab treatment was 86 (range: 1-869) days.

\section{Third-line treatment patterns}

A median interval of 28 (range: 1-1021) days passed between second- and third-line therapy, and the median duration of third-line therapy was 59 (range: 1-1085) days. The most common third-line regimens were nivolumab (38.4\%), gemcitabine (17.8\%) and docetaxel (13.0\%) (Table 3). The median duration of nivolumab treatment was 75 (range: 1-840) days. 
Table 1. Baseline patient demographics and clinical characteristics.

\section{Variable}

Age at index, years:

$$
\text { - Mean (SD) }
$$

- Median (range)

Sex, n (\%):

- Male

- Female

- Missing

Race/ethnicity, $\mathrm{n}(\%)$ :

- Asian
- Black or African-American
- Hispanic or Latino
- White
- Other
- Missing
Geographic region of residence in the USA, $n(\%):$

- Northeast
- Midwest

- South 3199

- West

- Missing

Smoking history, $\mathrm{n}(\%)$ :

- History of smoking

- No history of smoking

- Unknown/not documented

$\mathrm{BMI}, \mathrm{kg} / \mathrm{m}^{2}$ :

- Mean (SD)

- Median (range)

Days from initial NSCLC diagnosis to index date, $n(\%)$ :

\begin{tabular}{ll} 
- Mean (SD) & $14.6(83.5)$ \\
\hline- Median (range) & $0(0-1066)$ \\
\hline Stage of cancer at initial diagnosis, $n(\%)^{\dagger}:$ & $99(1.0)$ \\
\hline- I & $79(0.8)$ \\
- II & $153(1.6)$ \\
\hline - IIIA & $0(0.0)$ \\
\hline - IIIB & $9287(96.2)$ \\
\hline - IV & $38(0.4)$ \\
\hline - Missing & \\
\hline Concomitant drugs, $n(\%)^{\ddagger}:$ & $20(0.2)$ \\
\hline - Antianemics & $186(1.9)$ \\
\hline - Antiemetics & $57(0.6)$ \\
\hline - Anti-infectives & $33(0.3)$ \\
\hline - Bone-modifying agents & $64(0.7)$ \\
\hline - Granulocyte colony-stimulating factors & $103(1.1)$ \\
\hline - Pain agents & $215(2.2)$ \\
\hline - Solution fluids & $8(0.08)$ \\
\hline - Steroids &
\end{tabular}

185 (1.9)

738 (7.6)

223 (2.3)

6012 (62.3)

$900(9.3)$

1598 (16.5)

2086 (21.6)

1886 (19.5)

3199 (33.1)

1364 (14.1)

1121 (11.6)

8519 (88.2)

920 (9.5)

217 (2.2)

25.9 (5.6)

25 (13-65)

4.6 (83.5)

(0-1066)

99 (1.0)

$9(0.8)$

$53(1.6)$

287 (96.2)

8 (0.4)

$0(0.2)$

$86(1.9)$

$33(0.3)$

$64(0.7)$

$215(2.2)$

$\dagger$ Initial diagnosis could have occurred prior to the index date, and all patients had evidence of metastatic disease by the index date.

$¥$ Patients could receive $>1$ concomitant drug.

$\S \%$ calculated from documented cases.

I Patients with recorded positive test results for EGFRm or ALK rearrangements were excluded from the study.

ECOG PS: Eastern Cooperative Oncology Group performance status; EGFRm: EGFR mutation; KRASm: KRAS mutation; N/A: Not applicable; NSCLC: Non-small-cell lung cancer; SD:

Standard deviation. 
Table 1. Baseline patient demographics and clinical characteristics (cont.).

\begin{tabular}{|c|c|}
\hline Variable & Metastatic NSCLC $(n=9656)$ \\
\hline \multicolumn{2}{|c|}{ ECOG PS at index date, $\mathrm{n}(\%)^{\S}$ : } \\
\hline-0 & $250(41.9)$ \\
\hline-1 & $231(38.7)$ \\
\hline-2 & $99(16.6)$ \\
\hline-3 & $17(2.8)$ \\
\hline-4 & $0(0.0)$ \\
\hline \multicolumn{2}{|c|}{ Site of metastases, $n(\%)^{\S}$ : } \\
\hline - Brain & N/A \\
\hline - Liver & $54(11.9)$ \\
\hline - Bone & $239(53.0)$ \\
\hline - Other & $158(35.0)$ \\
\hline \multicolumn{2}{|l|}{ EGFRm, $\mathrm{n}(\%) \mathbb{I}:$} \\
\hline - Yes & N/A \\
\hline$-\mathrm{No}$ & $116(1.2)$ \\
\hline - Not tested & 9540 (98.8) \\
\hline \multicolumn{2}{|c|}{$A L K$ rearrangements, $\mathrm{n}(\%) \mathbb{I}:$} \\
\hline - Yes & N/A \\
\hline$-\mathrm{No}$ & $106(1.1)$ \\
\hline - Not tested & $9550(98.9)$ \\
\hline \multicolumn{2}{|l|}{ KRASm, n (\%): } \\
\hline \multicolumn{2}{|c|}{$\begin{array}{l}\text { †nitial diagnosis could have occurred prior to the index date, and all patients had evidence of metastatic disease by the index date. } \\
\text { ‡Patients could receive }>1 \text { concomitant drug. } \\
\$ \% \text { calculated from documented cases. } \\
\text { I Patients with recorded positive test results for EGFRm or ALK rearrangements were excluded from the study. } \\
\text { ECOG PS: Eastern Cooperative Oncology Group performance status; EGFRm: EGFR mutation; KRASm: KRAS mutation; N/A: Not applicable; NSCLC: Non-small-cell lung cancer; SD: } \\
\text { Standard deviation. }\end{array}$} \\
\hline
\end{tabular}

\section{Summary of treatment patterns}

During the follow-up period, the median number of systemic treatment lines among patients with at least one regimen was 1 (range: $0-8$ ). The median overall duration of treatment in patients with recorded treatments was 174 (range: 1-1665) days. Overall, 6455 (66.8\%) patients received first-line therapy, 2966 (30.7\%) received second-line therapy, and $1204(12.5 \%)$ received third-line therapy. Among patients with recorded treatments, $64.1 \%$ received chemotherapy, $21.1 \%$ received targeted therapy or an antiangiogenic agent, and $18.0 \%$ received immunotherapy; more than one quarter $(26.7 \%)$ of patients had no recorded systemic treatments. In summary, carboplatin + paclitaxel $(18.6 \%)$ was the most common first-line therapy, while nivolumab was the most commonly prescribed second- and third-line therapy (31.0 and 38.4\%, respectively; Table 3).

Overall survival

At $64.1 \%$ data maturity, median OS was 11.1 months (95\% CI: 10.8-11.5; Figure 2A) from the index date, 
Table 2. Select clinical characteristics during follow-up ${ }^{\dagger}$.

\section{Clinical characteristic}

Duration of treatment (days):

$$
\text { - Mean (SD) }
$$$$
\text { - Median (range) }
$$

Concomitant drugs, $\mathrm{n}(\%)^{\ddagger}$ :

\begin{tabular}{l} 
- Antianemics \\
\hline - Antiemetics \\
- Anti-infectives \\
\hline - Bone-modifying agents
\end{tabular}

\section{- Bone-modifying agents}

- Granulocyte colony-stimulating factors

- Pain agents

- Solution fluids

- Steroids

ECOG PS $<60$ days after index date, $\mathrm{n}(\%)^{\S}$ :

$-0$

$-1$

$-2$

$-3$

$-4$

- Not documented

Site of metastases, $\mathrm{n}(\%)^{\S}$ :

- Brain

- Liver

- Bone

- Other

- Not documented

EGFRm, $\mathrm{n}(\%) \mathbb{I}$.

- Yes

- No

- Not tested

ALK rearrangements, $\mathrm{n}(\%) \mathbb{I}$ :

- Yes

- No

- Not tested

KRASm, n (\%):

- Yes

- No

- Not tested

PD-L1 expression, $n(\%)$ :

- Positive

- Negative

- Not tested
Metastatic NSCLC $(n=9656)$

$287.4(302.9)$
$174(1-1665)$

1208 (12.5)

6202 (64.2)

462 (4.8)

2017 (20.9)

3019 (31.3)

909 (9.4)

4041 (41.8)

224 (2.3)

1161 (32.3)

1413 (39.3)

738 (20.5)

271 (7.5)

$17(0.5)$

6056 (62.7)

1064 (23.6)

382 (8.5)

2082 (46.2)

974 (21.6)

5154 (53.4)

N/A

4870 (50.4)

4786 (49.6)

$\mathrm{N} / \mathrm{A}$

4625 (47.9)

5031 (52.1)

595 (6.2)

1020 (10.6)

8041 (83.3)

233 (2.4)

315 (3.3)

9108 (94.3)

$\dagger$ Follow-up defined as the time from the index date to the earliest date of either death or the last visit in the database prior to or at the end of the data collection period (31 January 2017).

$\ddagger$ Patients could receive $>1$ concomitant drug.

$\S \%$ calculated from documented cases.

I Patients with recorded positive test results for EGFRm or ALK rearrangements were excluded from the study.

ECOG PS: Eastern Cooperative Oncology Group performance status; EGFRm: EGFR mutation; KRASm: KRAS mutation; N/A: Not applicable; NSCLC: Non-small-cell lung cancer; SD: Standard deviation. 


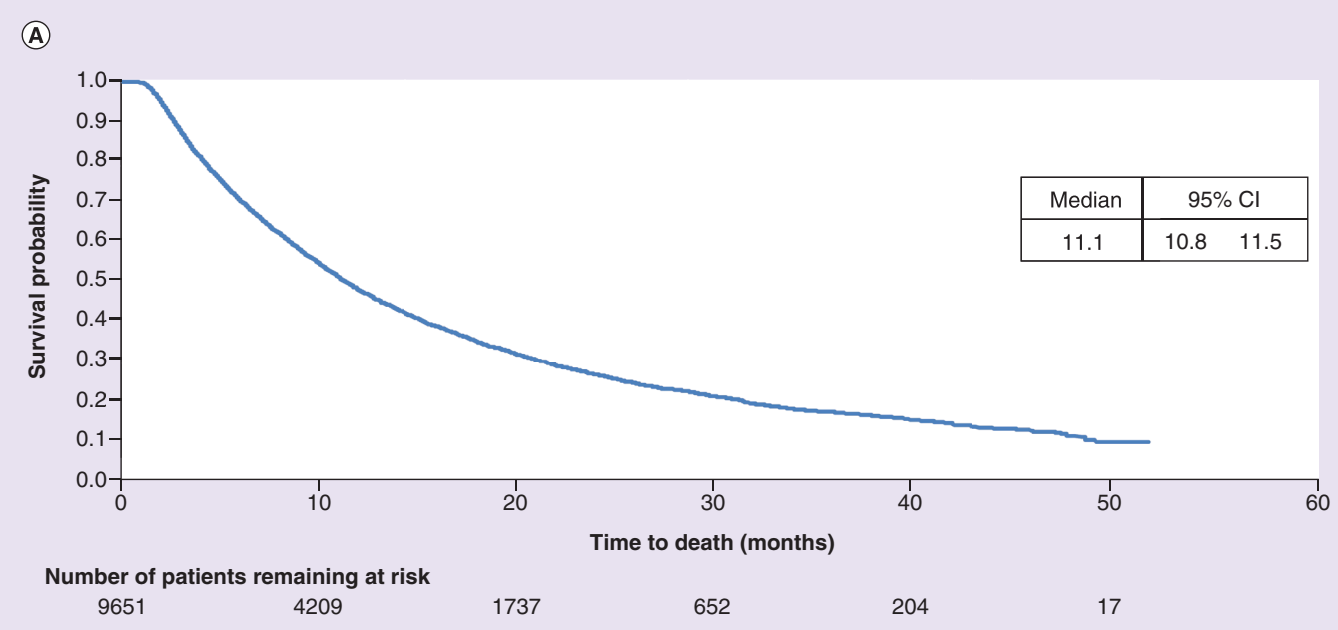

(B)

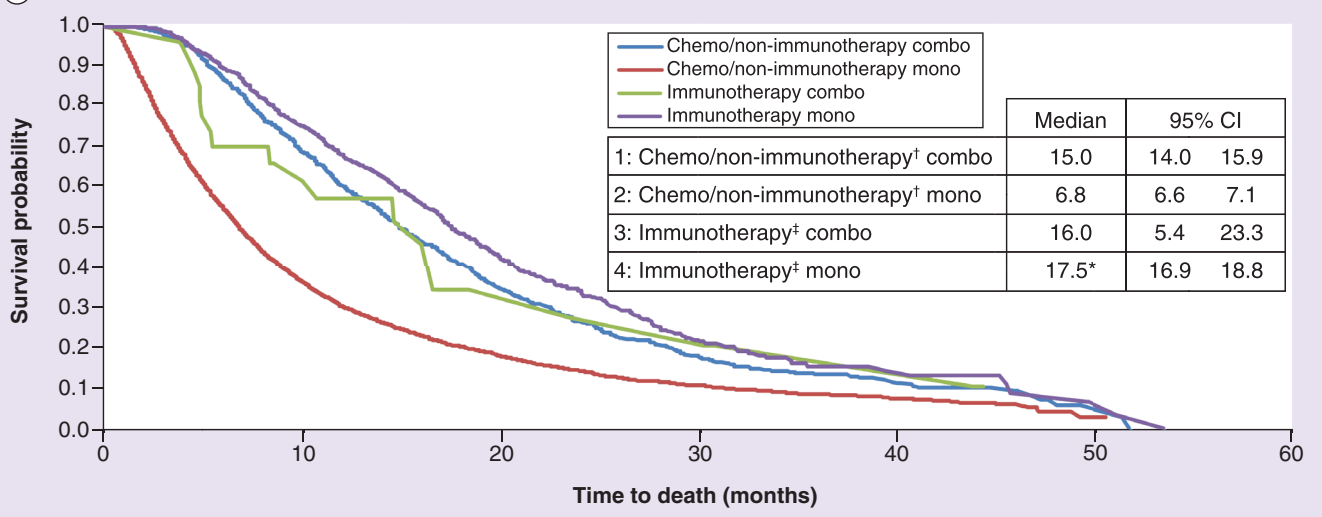

Number of patients remaining at risk

$\begin{array}{llccccc}1 & 1045 & 673 & 284 & 108 & 33 & 2 \\ 2 & 5183 & 1580 & 554 & 214 & 67 & 1 \\ 3 & 27 & 14 & 5 & 4 & 2 & 0 \\ 4 & 1073 & 769 & 279 & 57 & 13 & 2\end{array}$

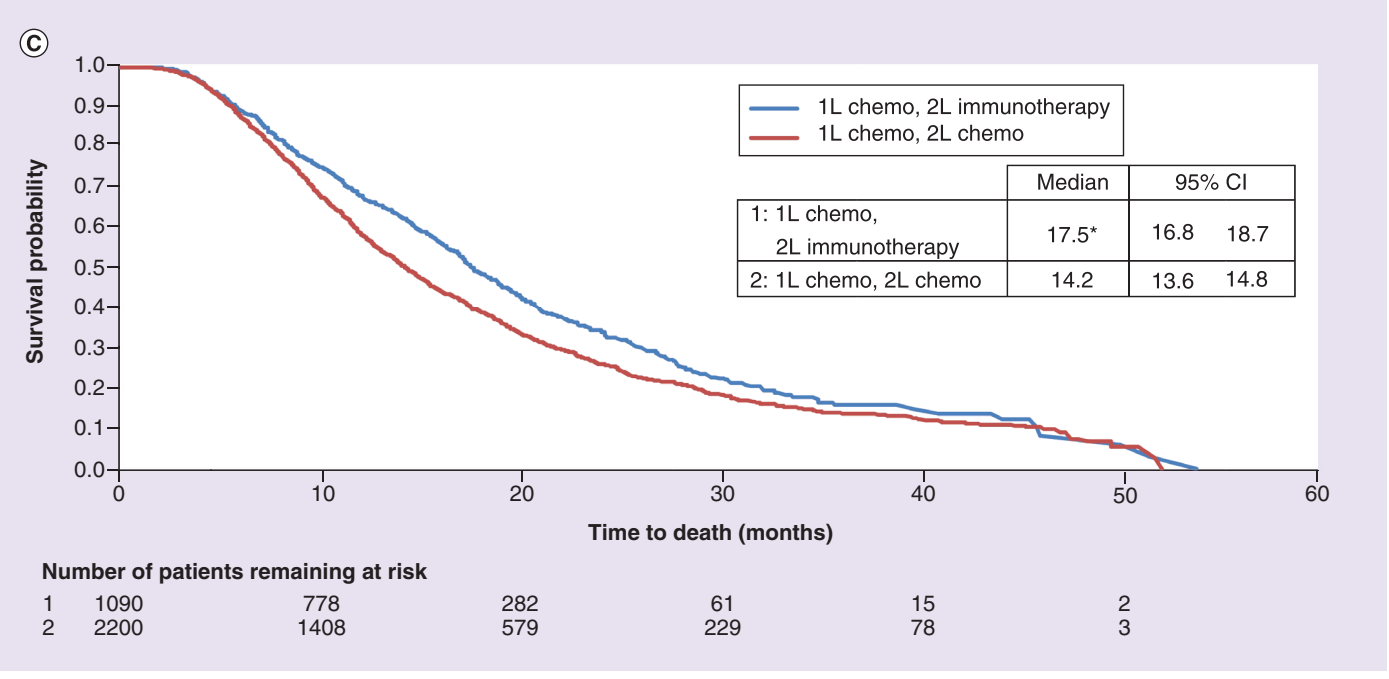

Figure 2. Overall survival. Overall survival of patients with metastatic non-small-cell lung cancer not previously treated with systemic therapy at baseline (A) from index date; (B) by first-line therapy type; (C) by treatment sequence.

${ }^{*} \mathrm{p}<0.05$ versus comparator.

†Chemo/nonimmunotherapy defined as platinum chemotherapy (cisplatin and carboplatin), other chemotherapy (gemcitabine, docetaxel, paclitaxel, pemetrexed and vinorelbine), targeted therapy (ceritinib, crizotinib, erlotinib, gefitinib, osimertinib and other agents) or antiangiogenic therapy (bevacizumab and ramucirumab).

¥Immunotherapy defined as ipilimumab, nivolumab, pembrolizumab or atezolizumab.

1L: First line; 2L: Second line; Chemo: Chemotherapy; Combo: Combination; Mono: Monotherapy; NSCLC: Non-small-cell lung cancer; OS: Overall survival. 


\begin{tabular}{|c|c|}
\hline Top five first-line regimens received, $\mathrm{n}(\%)$ & Patients with recorded first-line treatment $(n=6455)$ \\
\hline Carboplatin + paclitaxel & $1201(18.6)$ \\
\hline Carboplatin + pemetrexed & $1071(16.6)$ \\
\hline Bevacizumab + carboplatin + pemetrexed & $727(11.3)$ \\
\hline Carboplatin + nab-paclitaxel & $494(7.7)$ \\
\hline Bevacizumab + carboplatin + paclitaxel & $447(6.9)$ \\
\hline Top five second-line regimens received, $\mathrm{n}(\%)$ & Patients with recorded second-line treatment $(n=2966)$ \\
\hline Nivolumab & $920(31.0)$ \\
\hline Pemetrexed & $278(9.4)$ \\
\hline Docetaxel & $250(8.4)$ \\
\hline Gemcitabine & $175(5.9)$ \\
\hline Erlotinib & $108(3.6)$ \\
\hline Top five third-line regimens received, $\mathrm{n}(\%)$ & Patients with recorded third-line treatment $(n=1204)$ \\
\hline Nivolumab & $462(38.4)$ \\
\hline Gemcitabine & $214(17.8)$ \\
\hline Docetaxel & $156(13.0)$ \\
\hline Vinorelbine & $134(11.1)$ \\
\hline Docetaxel + ramucirumab & $120(10.0)$ \\
\hline
\end{tabular}

11.7 months (95\% CI: 11.3-12.0) from the initial date of NSCLC diagnosis, and 10.1 months (95\% CI: 9.7-10.4) from the start of first-line therapy. Median OS was longer with first-line immunotherapy (17.5 months [95\% CI: 16.9-18.8]; $60.6 \%$ data maturity) versus chemotherapy/nonimmunotherapy combination therapy (15 months [95\% CI: $14.0-15.9$ ]; $75.6 \%$ data maturity; $\mathrm{p}<0.05$ ) and chemotherapy/nonimmunotherapy monotherapy (6.8 months [95\% CI: 6.6-7.1]; 77.3\% data maturity; $\mathrm{p}<0.05$; Figure 2B). Median OS was 17.5 months (95\% CI: $16.8-18.7 ; 60.8 \%$ data maturity) with first-line chemotherapy and second-line immunotherapy, and was longer compared with first- and second-line chemotherapy (14.2 months [95\% CI: 13.6-14.8]; 76.1\% data maturity; p $<0.05$; Figure 2C).

\section{Discussion}

Results of this retrospective, real-world, US community oncology-based study provide pertinent, new treatment pattern and survival data for patients with metastatic NSCLC. Nearly all patients were initially diagnosed with metastatic NSCLC, and, among patients with recorded treatments, approximately $64 \%$ received chemotherapy, $21 \%$ received targeted therapy or antiangiogenic therapy, and $18 \%$ received immunotherapy across all treatment lines. More than one quarter $(26.7 \%)$ of patients had no recorded systemic treatments, and the median OS from diagnosis was $<1$ year.

Most patients received first-line platinum-based chemotherapy consistent with both clinical guidelines and other real-world studies conducted during a similar time period [6-8,29]. Notably, the current study observed treatment patterns related to the approval and uptake of immunotherapy into clinical practice from 2015 onwards; in the second line of treatment, we observed that nivolumab was initiated after first-line platinum-based chemotherapy in nearly a third of patients. A retrospective analysis reports that $>60 \%$ of eligible patients with NSCLC received nivolumab within 4 months of US FDA approval [28], and the current data support these findings. Owing to the study time frame and the low initial use of pembrolizumab in the USA $[27,28]$, pembrolizumab use was rarely captured in this study; it was prescribed to only $1.4 \%$ of patients across all treatment lines. For the second-line treatment of metastatic NSCLC with nivolumab, PD-L1 testing is not required with the US FDA-approved complementary diagnostic test Dako PD-L1 28-8 pharmDx [13]. Consequently, we observed a low rate of PD-L1 testing during the study period, which was also likely related to the low use of pembrolizumab. The approval of pembrolizumab as first-line therapy and its survival benefit over chemotherapy [12,15 are likely to drive PD-L1 testing in the future as immunotherapy becomes more common place in the treatment of metastatic NSCLC.

With regard to treatment with targeted therapies, we observed the use of erlotinib in the second line of treatment. The US FDA label update limiting erlotinib to the treatment of patients with EGFRm metastatic NSCLC occurred in October 2016 [30]. Therefore, the treatment patterns observed in the current study reflect the US FDA approval 
erlotinib received from 2004 onwards as monotherapy for the treatment of patients with metastatic NSCLC, regardless of genetic mutation status, after failure of at least one prior chemotherapy regimen.

The median OS observed in this study (11.1 months from metastatic NSCLC diagnosis and 10.1 months from the start of first-line therapy) was generally consistent with reports in the literature [8,29]. We observed a significant survival benefit with first-line immunotherapy monotherapy compared with first-line chemotherapy/nonimmunotherapy combination therapy and monotherapy (median OS: 17.5 vs 15 months and 6.8 months, respectively). As pembrolizumab use was low, it is likely that nivolumab was used off-label as first-line immunotherapy. In real-world practice, a substantial number of patients receive off-label immunotherapy treatment [31], and our current findings reflect this. Following first-line chemotherapy, survival was longer with second-line immunotherapy compared with second-line chemotherapy (median OS: 17.5 vs 14.2 months, respectively). Continued research to better understand the benefits of first- and second-line immunotherapy in these patients, particularly for subgroups in which these medications might be particularly effective, is needed. Indeed, our analysis was unable to capture treatment with recently approved immunotherapies, particularly in the first-line setting, and future real-world studies are needed to address this. Furthermore, while current PD-L1 testing rates are low, future real-world studies may be able to distinguish efficacy by PD-L1 expression level.

There are several limitations of this study. Broadly, we found that replicating a clinical trial population in a real-world setting was difficult due to the extent of missing clinical information. For example, the Flatiron database was a convenience sample that lacked complete entries for many clinical variables (e.g., ECOG PS) that are not routinely recorded by clinicians. Information on the site of metastasis was not available for the majority of patients at baseline. Data regarding comorbidities treated and medications prescribed outside of the oncology clinic setting were under-recorded in the database. Information on treatment provided in hospitals (e.g., surgery and radiation) or other settings was not available, which may have resulted in misclassification of treatment and outcomes. As the stage of disease was not available in the Flatiron cohort of patients with advanced NSCLC, we relied on a documented initial diagnosis of metastatic disease for patient selection, and, for patients whose disease had progressed from an earlier stage to metastatic disease, ICD-10 codes indicating secondary tumors were used, which were under-reported in the database. As a result, most patients included in the study were initially diagnosed with metastatic NSCLC, and patients with disease progression from an earlier stage to metastatic disease were under-represented. Therefore, generalization of the current results to patients who are diagnosed with NSCLC at an earlier stage and progress to metastatic disease should be made with caution. Additionally, the analyses of OS by treatment sequence, and OS from the initial NSCLC diagnosis date, included immortal time for some patients: a period of follow-up during which death could not occur, as patients must have survived long enough to receive at least two lines of therapy, and, when the initial NSCLC diagnosis date preceded the index date, patients must have survived from the initial NSCLC diagnosis date to the index date [32].

\section{Conclusion}

In this retrospective, real-world, US community oncology-based study of patients with metastatic NSCLC, median OS was $<1$ year from the diagnosis of metastatic disease. More than one quarter of the patients had no

\section{Summary points}

- Following the approval of immunotherapy for patients with metastatic non-small-cell lung cancer (NSCLC), there is an unmet need for real-world evidence documenting treatment patterns and survival outcomes.

- This retrospective, observational study used electronic medical records to assess the treatment patterns and overall survival of over 9000 patients with metastatic NSCLC treated in real-world settings in the USA.

- Among patients with recorded treatments, approximately $64 \%$ received chemotherapy, $21 \%$ received targeted therapy/antiangiogenic agents and $18 \%$ received immunotherapy.

- Over one quarter of the patients had no recorded systemic treatments after diagnosis.

- Platinum chemotherapy was the most common first-line therapy, while immunotherapy was the most common second-line and third-line treatment.

- The median overall survival of patients from the initial diagnosis of metastatic disease was 11.1 months.

- A median overall survival benefit of over 3 months was observed with second-line immunotherapy versus second-line chemotherapy.

- Continued research is required to understand the survival benefit offered by immunotherapy for patients with metastatic NSCLC. 
recorded systemic treatments after diagnosis during a median follow-up of 8.3 months. Rates of PD-L1 testing were low, and many clinical characteristics were under-reported or missing. Platinum-based chemotherapy was most commonly administered as first-line treatment. Following US FDA approval in 2015, rapid uptake of immunotherapy into clinical practice was observed; nivolumab monotherapy was the most commonly prescribed second-line and later treatment. First-line immunotherapy was associated with a survival benefit relative to first-line chemotherapy/nonimmunotherapy, and median OS was longer with second-line immunotherapy compared with second-line chemotherapy. Therefore, it is important to develop new therapies, including immunotherapies and combination strategies, to broaden the population of patients with metastatic NSCLC that can access and benefit from treatment.

Financial \& competing interests disclosure

This work was supported by AstraZeneca. The sponsor was involved in the study design; collection, analysis and interpretation of data; report writing; and the decision to submit. J Simeone and B Nordstrom are employees of Evidera, and Evidera received funding from AstraZeneca for this study. A Klein is an employee and stockholder of AstraZeneca. K Patel is a previous employee of AstraZeneca. The authors have no other relevant affiliations or financial involvement with any organization or entity with a financial interest in or financial conflict with the subject matter or materials discussed in the manuscript apart from those disclosed.

Medical writing support, which was in accordance with Good Publication Practice (GPP3) guidelines, was provided by L Gillies, of Cactus Communications and was funded by AstraZeneca.

\section{Ethical conduct of research}

The authors confirm that all study data were fully compliant with US patient confidentiality requirements, including the Health Insurance Portability and Accountability Act (HIPAA) of 1996. The study used only de-identified patient records and, therefore, was exempted from Institutional Review Board approval. Informed consent was not required as this was not an interventional study, and routinely collected, anonymized data were used.

\section{Open access}

This work is licensed under the Attribution-NonCommercial-NoDerivatives 4.0 Unported License. To view a copy of this license, visit http://creativecommons.org/licenses/by-nc-nd/4.0/

\section{References}

Papers of special note have been highlighted as: • of interest; $\bullet \bullet$ of considerable interest

1. American Cancer Society. About non-small-cell lung cancer (2018). www.cancer.org/content/dam/CRC/PDF/Public/8703.00.pdf

2. Kratzke R, Franklin MJ. Lung cancer epidemiology. In: Encyclopedia of Cancer. Schwab M (Ed.). Springer, Berlin, Heidelberg, Germany (2011).

3. Pikor LA, Ramnarine VR, Lam S, Lam WL. Genetic alterations defining NSCLC subtypes and their therapeutic implications. Lung Cancer 82(2), 179-189 (2013).

4. Noone AM, Howlader N, Krapcho M et al. SEER Cancer Statistics Review, 1975-2015 (2018). https://seer.cancer.gov/csr/1975_2015/

5. American Cancer Society. Non-small-cell lung cancer survival rates, by stage (2018). www.cancer.org/cancer/non-small-cell-lung-cancer/detection-diagnosis-staging/survival-rates.html

6. Hanna N, Johnson D, Temin S et al. Systemic therapy for stage IV non-small-cell lung cancer: American Society of Clinical Oncology clinical practice guideline update. J. Clin. Oncol. 35(30), 3484-3515 (2017).

7. Novello S, Barlesi F, Califano R et al. Metastatic non-small-cell lung cancer: ESMO clinical practice guidelines for diagnosis, treatment and follow-up. Ann. Oncol. 27(suppl 5), v1-v27 (2016).

8. Abernethy AP, Arunachalam A, Burke T et al. Real-world first-line treatment and overall survival in non-small-cell lung cancer without known EGFR mutations or ALK rearrangements in US community oncology setting. PLoS ONE 12(6), e0178420 (2017).

-. The results establish a baseline for care and overall survival based upon contemporary first-line treatments for patients with metastatic non-small-cell lung cancer treated in the US community oncology setting.

9. Santana-Davila R, Szabo A, Arce-Lara C, Williams CD, Kelley MJ, Whittle J. Cisplatin versus carboplatin-based regimens for the treatment of patients with metastatic lung cancer. An analysis of Veterans Health Administration data. J. Thorac. Oncol. 9(5), 702-709 (2014).

10. Ezeife DA, Leighl NB. Personalized medicine for non-small-cell lung cancer: where are we now and where can we go? Expert Rev. Respir. Med. 12(2), 81-82 (2018).

11. Postow MA, Callahan MK, Wolchok JD. Immune checkpoint blockade in cancer therapy. J. Clin. Oncol. 33(17), 1974-1982 (2015). 
12. Keytruda ${ }^{\circledR}$, prescribing information. Merck Sharp \& Dohme, NJ, USA. www.merck.com/product/usa/pi_circulars/k/keytruda/keytruda_pi.pdf

13. Opdivo ${ }^{\circledR}$, prescribing information. Bristol-Myers Squibb, NJ, USA. https://packageinserts.bms.com/pi/pi_opdivo.pdf

14. Tecentriq ${ }^{\circledR}$, prescribing information. Genentech Inc, CA, USA. www.gene.com/download/pdf/tecentriq-prescribing.pdf

15. Reck M, Rodríguez-Abreu D, Robinson AG et al. Pembrolizumab versus chemotherapy for PD-L1-positive non-small-cell lung cancer. N. Engl. J. Med. 375, 1823-1833 (2016).

- The Phase III KEYNOTE-024 trial demonstrates the improved efficacy and safety of immunotherapy versus platinum-based chemotherapy for patients with untreated, advanced non-small-cell lung cancer with PD-L1 expression $\geq \mathbf{5 0 \%}$.

16. Antonia S, Goldberg SB, Balmanoukian A et al. Safety and antitumour activity of durvalumab plus tremelimumab in non-small-cell lung cancer: a multicentre, Phase 1b study. Lancet Oncol. 17(3), 299-308 (2016).

17. Hellmann MD, Ciuleanu TE, Pluzanski A et al. Nivolumab plus ipilimumab in lung cancer with a high tumor mutational burden. $N$. Engl. J. Med. 378(22), 2093-2104 (2018).

18. Peters S, Antonia S, Goldberg SB et al. 191TiP: MYSTIC: a global, Phase 3 study of durvalumab (MEDI4736) plus tremelimumab combination therapy or durvalumab monotherapy versus platinum-based chemotherapy (CT) in the first-line treatment of patients (pts) with advanced stage IV NSCLC. J. Thorac. Oncol. 11(Suppl. 4), S139-S140 (2016).

19. AstraZeneca. Imfinzi demonstrates clinical activity in Stage IV, 1st-line non-small cell lung cancer in Phase III MYSTIC trial (2018). www.astrazeneca.com/media-centre/medical-releases/imfinzi-demonstrates-clinical-activity-in-stage-iv-1st-line-non-small-cell-lung-c ancer-in-phase-iii-mystic-trial-13122018.html

20. Rizvi N, Chul Cho B, Reinmuth N et al. LBA6 - Durvalumab with or without tremelimumab vs platinum-based chemotherapy as first-line treatment for metastatic non-small cell lung cancer: MYSTIC. Ann. Oncol. 29(Suppl. 10), x39-x43 (2018).

21. Peters S, Chul Cho B, Reinmuth N et al. Tumor mutational burden (TMB) as a biomarker of survival in metastatic non-small cell lung cancer (mNSCLC): blood and tissue TMB analysis from MYSTIC, a Phase III study of first-line durvalumab \pm tremelimumab vs chemotherapy [abstract]. Cancer Res. 79(Suppl. 13), Abstract CT074 (2019).

22. Planchard D, Yokoi T, McCleod MJ et al. A Phase III study of durvalumab (MEDI4736) with or without tremelimumab for previously treated patients with advanced NSCLC: rationale and protocol design of the ARCTIC study. Clin. Lung Cancer 17(3), 232-236 (2016).

23. Mok T, Schmid P, Arén O et al. 192TiP: NEPTUNE: a global, Phase 3 study of durvalumab (MEDI4736) plus tremelimumab combination therapy versus standard of care ( $\mathrm{SoC}$ ) platinum-based chemotherapy in the first-line treatment of patients (pts) with advanced or metastatic NSCLC. J. Thorac. Oncol. 11(Suppl. 4), S140-S141 (2016).

24. Mok T, Johnson M, Garon E et al. P1.04-008 POSEIDON: A Phase 3 study of first-line durvalumab \pm tremelimumab+chemotherapy vs chemotherapy alone in metastatic NSCLC. J. Thorac. Oncol. 12(Suppl. 2), S1975 (2017).

25. Garassino MC, Cho BC, Kim JH et al. Durvalumab as third-line or later treatment for advanced non-small-cell lung cancer (ATLANTIC): an open-label, single-arm, Phase 2 study. Lancet Oncol. 19(4), 521-536 (2018).

26. Flatiron Health. https://flatiron.com/publications/

27. Khozin S, Abernethy AP, Nussbaum NC et al. Characteristics of real-world metastatic non-small-cell lung cancer patients treated with nivolumab and pembrolizumab during the year following approval. Oncologist 23(3), 328-336 (2018).

-. The multicenter analysis used electronic medical record data from the Flatiron database to capture the use of PD-1 inhibitors as they first entered clinical use in the USA for the treatment of patients of metastatic non-small-cell lung cancer.

28. O'Connor JM, Fessele KL, Steiner J et al. Speed of adoption of immune checkpoint inhibitors of programmed cell death 1 protein and comparison of patient ages in clinical practice vs pivotal clinical trials. JAMA Oncol. 4(8), e180798 (2018).

-. The retrospective cohort study uses electronic medical record data from the Flatiron database to assess the speed with which anti-PD-1 agents reached eligible patients in practice, and to compare the ages of patients treated in clinical practice versus those treated in clinical trials.

29. Nadler E, Espirito JL, Pavilack M, Boyd M, Vergara-Silva A, Fernandes A. Treatment patterns and clinical outcomes among metastatic non-small-cell lung cancer patients treated in the community practice setting. Clin. Lung Cancer 19(4), 360-370 (2018).

- A retrospective, observational cohort study that describes real-world treatment and survival in patients with metastatic non-small-cell lung cancer.

30. Tarceva ${ }^{\circledR}$, prescribing information. Genentech and Astellas Pharma US, Genentech, CA, USA; Astellas, Tokyo, Japan. https://www.accessdata.fda.gov/drugsatfda_docs/label/2016/021743s025lbl.pdf

31. De Souza JA, Duong YY. Off-label immunotherapy prescription: financial implications for payers and patients. J. Clin. Oncol. 35(Suppl. 8), 6-6 (2017).

32. Suissa S. Immortal time bias in pharmacoepidemiology. Am. J. Epidemiol. 167(4), 492-499 (2008).

- Describes the immortal time bias and shows how it can occur in observational studies of medication effects under a variety of cohort designs. 